CYBERNETICS AND INFORMATION TECHNOLOGIES • Volume 16, No 5

Special Issue on Application of Advanced Computing and Simulation in Information Systems

Sofia $\bullet 2016$

\title{
Synchronous Rendezvous Based on Cluster in Low-Duty-Cycle Wireless Sensor Network
}

\section{Shuhong Cheng, Wenke Zhang}

Department of Information Engineering, Chongqing City Management College, Chongqing 401331, China

Emails: 279618605@qq.com103686741@qq.com

\begin{abstract}
This paper presents SRBC, a new design for synchronous rendezvous in low-duty-cycle Wireless Sensor Network (WSN).The main idea of SRBC is utilizing the cluster, which includes a part of the sensor nodes in the WSN. Each node in a cluster alters its clock drift as well as its skew towards the central node of the cluster through exchange of their clock information with normal communication. Then it reduces the overhead of the process usual for the traditional time-stamp exchange. In different clusters, the border nodes exchange the relative clock drift as well as the skew to improve the performance during the synchronous rendezvous. Results show that the design of SRBC is practical and effective.
\end{abstract}

Keywords: Wireless sensor network, synchronous rendezvous, low-duty-cycle, clock drift, clock skew.

\section{Introduction}

Recent advances in wireless communication technologies, embedded computing, sensor technology, Micro-Electro-Mechanical Systems (MEMS) technology make Wireless Senor Networks (WSNs) to show a rapid growth in development. WSNs, made up of a large number of low-cost, low-power, and multi-function sensor nodes, are designed for monitoring tasks, such as battlefield surveillance, equipment supervision, intruder detection, and wildlife observation. Usually, sensor nodes in a WSN are battery-powered and it is difficult to recharge or replace batteries in harsh or hostile environment. It is well known that keeping nodes in sleep mode, i.e., letting them operate in low-duty-cycle mode, can effectively save energy at the cost of increased communication delays. Therefore, how to achieve real-time data delivery in low-duty-cycle WSNs is a problem which worth to be investigated [1-2]. This brings about the problem that when a node needs to send message to its 
neighbour node, the neighbour node might be not just in its work slot, while the rendezvous before the communication need the two nodes to be in the work state [3]. It's impossible for nodes which need to communicate with each other to be all in their work slot in low-duty-cycle wireless sensor network; for each node its work slot is extremely short in comparison to its work cycle. Then, it's necessarily to find a way for the node which needs to send message to work in the same time with the node which will receive this message. Theoretically, if all nodes have the same clock, this process is just the synchronous rendezvous; for example, node $A$ needs to communicate with Node $B$, node $A$ has node $B$ 's work cycle, then node $A$ just awakes during the node $B$ 's work slot to establish a communication link. This is easy to be utilized in almost all MAC layer protocol, to improve the performance of energy efficiency greatly without any change of the initial design.

But each node has its own clock, which has different clock skew [4]; with the increase of working time, a slightly relative skew could bring larger clock drift, then node $A$ couldn't predict node $B$ 's work slot according to its own clock. It's important for node $A$ to predict the relative clock drift comparing with node $B$, and then to get the real work slot of node $B$. If the appropriate method is just working without increasing the additional traffic, while satisfied that each node which needs to send message could predict the work slot of the node which will receive the message from node $A$ (in fact the two nodes are just neighbor nodes), that means, the estimation of node $B$ 's clock by node $A$ has sufficiently small relative clock error. Then node $A$ could achieve its own clock and the work cycle of node $B$; then node $A$ begins to synchronize rendezvous process with the node $B$ according to the communication process successfully, and thereby improving the efficiency of the network. Of course, this synchronous rendezvous process only has little node energy consumption, thereby improves the network lifetime.

This paper focuses on improving the performance of synchronous rendezvous in the low-duty-cycle wireless sensor network with the existence of relative clock skew in each node. The algorithm improves the effectiveness and the reliability of the synchronous rendezvous through sharing the clock information in a cluster as well as among the clusters, to reduce estimated clock error based on the normal communication. The main work is organized as follows:

1) Detecting neighbor nodes' relative clock skew in comparison to a reference clock in a cluster based on the normal communication in low-duty-cycle WSN, as well as sharing the clock information to reduce the clock error of estimation.

2) Achieving synchronous rendezvous based on the clock estimation to improve the efficiency as well as the network lifetime.

In the following, we start with the related works in Section 2. Section 3 gives our design of SRBC, Section 4 reports simulation evaluation results.

\section{Related works}

Currently there are two types of rendezvous in low-duty-cycle Wireless Sensor Network (WSN): asynchronous rendezvous and synchronous rendezvous [5]. In a linear network, Dynamic Programming (DP) can be used to recursively augment the 
node's active slot so that an optimized solution can be obtained. However, in a complex network with multiple crossing paths, the DP becomes an NP-hard problem [6]. It is impossible for nodes which need to communicate with each other to be all in their work slot in low-duty-cycle wireless sensor network; for each node the work slot is extremely short compared to its work cycle. Then it is necessarily to find a way for the node which needs to send message to work in the same time with the node which will receive the message.

Through system modeling, Ye, Silva and Heidemann [7] formulate a multi-objective optimization problem, which augments minimal number of active slots and keeps the end-to-end delay within the delay bound. In addition, an optimization algorithm based on Non-dominated Sorting Genetic Algorithm (NSGA-II) is presented to solve the problem. Meanwhile, the idea of immune is introduced into the algorithm, which is realized by eliminating the initial active slot of each node before the genetic operation phase; this can guarantee that each node has at least one active slot in a duty cycle. Simulation results show that, in linear networks, the results obtained by the proposed algorithm are close to those obtained by DP. Furthermore, in complex networks, the proposed algorithm can remedy the shortcoming of DP and effectively solve the real-time delivery problem in low dutycycle sensor networks.

Ganeriwal et al. [8] propose a multi-pipeline feature based MultiSchedule Forwarding (MSF) strategy which includes two optimization algorithms. In this strategy, every node has more than one parent node and is able to assign different active times to all of them. In order to further reduce the time interval between data sensing and data receiving, we also take packet generation time into consideration while optimizing packet delivery latency. By assigning pre-calculated wake-up time slots to the nodes, the end-to-end average delay and maximum delay could be minimized through coordinating multiple paths with little costs. Secondly, Ganeriwal presented a Dynamic duty-cycle Control Strategy (D2CS) strategy based on opportunistic routing. In our design, nodes in network dynamically re-calculate their wake-up duration based on current packet latency. With D2CS, network delay can be controlled on demand and the overall duty-cycle could be reduced. Finally, the author compared the MSF strategy and D2CS strategy to traditional ones separately through experiments. The results show that with MSF strategy, both average and maximum end-to-end delay could be kept at a low level with very few overhead.

Chen et al. [9] verify and analyses typical opportunistic routing of the low duty-cycle WSN and mobile WSN based on probabilistic model checking technology separately; Chen improves these protocols. Detailed study contains three aspects as follows:

(1) Classical opportunistic routing of the low duty-cycle WSN is modeled, analyzed and verified by probabilistic model checking method. The verification result shows the problem of high rate of empty nodes. Aiming at this problem, the opportunistic routing with backtracking (LDCOP) has been designed to improve the problem of empty nodes. Its main idea is that if a next hop of minimization the remaining distance is not found during the maximum waiting time, the packet is not 
discarded, and the protocol allows the packet to initially move further away from the sink until a path to the destination is found. Then the tool model checking PRISM is used to check LDCOP and to prove that it has a better performance in packet forwarding rate and reducing empty nodes.

(2) Opportunistic routing of mobile WSN is modeled, analyzed and verified by probabilistic model checking method. The verified result shows the problem of short survival time. Aiming at this problem, Opportunistic routing based on energy aware and load balancing (PAGeRaF) is designed to solve the problem. This protocol improves the priority of candidate forwarding set. It joins the energy aware and load balancing. Then the tool PRISM is used to check PAGeRaF and it is found that the survival time of the network has been prolonged.

(3) The PAGeRaF is applied to VANET; the road density discovery and neighbor discovery mechanism are analyzed; the PAGeRaF based on VANET is simulated and analyzed using NS2inthe actual application scenarios, which shows that PAGeRaF can effectively reduce the number of network messages to avoid channel congestion compared with the traditional AODV routing.

The PSR [10] algorithm is the latest excellent algorithm used in low-dutycycle WSN. Its advantage is the correct detection of clock drift as well as the clock skew based on the normal communication, and the adaptive energy-driven mechanism to accommodate a variety of network traffic load. Of course, the most important advantage is that PSR could be utilized as a basic element of the MAC layer, it is easy to be utilized in almost all MAC layer protocol, to improve the performance of energy efficiency greatly without any change of the initial design. PSR algorithm provides an effective direction for synchronous rendezvous, which greatly saves node energy, thus extending the lifetime of the network. But PSR did not share the clock information with each other, nor establish a reference clock within a certain range (for example, a cluster), thereby the effectiveness of the rendezvous can be further improved, then saving more energy efficiency of the network.

\section{The SRBC algorithm}

Wireless sensor networks consist of autonomous sensor nodes that monitor the physical indicators of their environment and communicate with each other to transmit the data to monitoring centre. Wireless sensor networks inter fact the physical world with the digital domain, and they face both new application opportunities and new design challenges. The applications of sensor network include tracking and disturbance detection for military purposes, pollution and habitat monitoring for environmental purposes, warehouse storage and gas leak monitoring for industrial automation applications, traffic and location monitoring for civilian use. As specific application typically requires custom performance guarantees, so a wide range of applications create a design challenge in and of it. Today more than ever before, applications of sensor networks require individual nodes to reduce energy demand to prolong network life cycle. Each layer of the protocol stack must lower its own energy dissipation. Low-Power-Listening (LPL) 
protocols based on IEEE802.11 MAC protocol drastically reduce idle listening, a state of the node when its radio is in receive mode, but not receiving any packets. In a Low-Power-Listening protocol, nodes monitor the channel periodically, and if they do not receive any packets for a period of time, they return to sleep for another period of time. A low duty cycle is beneficial to receiving nodes, so they may return to sleep for longer periods of time, but at the same time, it may increase contention locally, thereby reducing the transmission of data packets. Energy efficiency is of the utmost importance in wireless sensor networks. Based on the analysis of the existing low-duty-cycle algorithms, here, we propose a new dynamic duty-cycle approach to control the duty-cycle for optimizing power consumption and balancing the load in sensor networks through predicting the load of next period. Both the analysis and simulation results show that our framework can appropriately adjust duty-cycle to the current network conditions, while the energy dissipation of idle listening is minimized. The results also reveal that our framework is more energy efficient than a fixed duty-cycle protocol.

For example, node $A$ and node $B$ work for time $t$, respectively, the time of each node is $t_{A}(t), t_{B}(t)$, then their relative difference $t_{\text {drift }}$ is the clock drift between them which is

$$
t_{\text {drift }}=t_{A}(t)-t_{B}(t) .
$$

Let's assume that the reference clock starts to work in time of $t_{0}$, after the time of $t_{\mathrm{R}}$, let's define the clock skew of node $n$ :

$$
S_{n}=\left(t_{n}-t_{\mathrm{R}}\right) / t_{\mathrm{R}} \text {. }
$$

\subsection{Establishing the reference clock}

Reference clock is established during the initialization time of the network, and changes dynamically during the work process. During the initialization time, each node sends its own clock information in turn to the center node in the cluster; the center node computes the average value of all the nodes, then broadcasts this average value with its timestamp to all the nodes; thereby all the nodes get their clock skews $S_{i}(t)$ toward the reference, while recording these information in their own clock information tables. The clock in different nodes can be seen from Fig. 1.

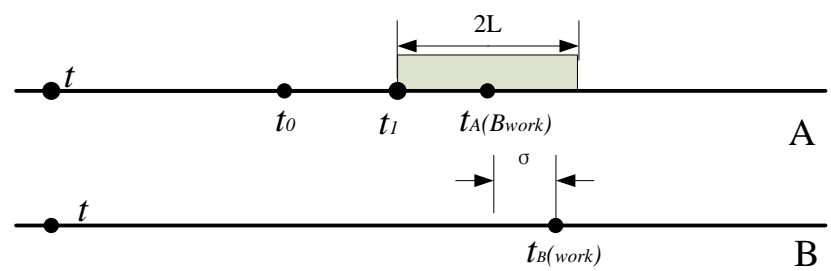

Fig. 1. The clock in different nodes

Node $A$ estimates the work time of node $B$ as $t_{A}\left(B_{\text {work }}\right)$, assumes that node $B$ has $n-1$ cycles (the cycle of node $B$ is $T_{B}$ ) from the calibration time $t_{B}(t)$ (the latest changing in $A$ 's clock information table), then: 


$$
t_{A}\left(B_{\text {work }}\right)=t_{B}(t)+n T_{B}\left(1+S_{B}(t)-S_{A}(t)\right) .
$$

It begins to work on the time of length $L$ to ensure the reliability of synchronous rendezvous, as shown in Fig. 1. Apparently the synchronous rendezvous could be achieved when node $B$ works during the time the node $A$ is working too, that:

$$
\mid t_{A}\left(B_{\text {work }}\right)-t_{B}(\text { work }) \mid \leq L .
$$

The new $t_{\text {wait }}$ is

$$
t_{\text {wait }}=t_{A}\left(t_{1}\right)-t_{A}\left(t_{0}\right) .
$$

It needs to be re-calibrated to improve the accuracy of the estimation when the error value is increased to a certain value in a cluster. In order to improve network efficiency, each node uses a calibration request while the error reaches a certain value (in our algorithm is $(2 / 3) L)$ during the normal communication, that is:

$$
\begin{cases}\left|t_{A}\left(B_{\text {work }}\right)-t_{B}\left(t_{1}\right)\right| \leq 2 L / 3, & I_{A}=\text { FALSE }, \\ \left|t_{A}\left(B_{\text {work }}\right)-t_{B}\left(t_{1}\right)\right|>2 L / 3, & I_{A}=\text { TRUE } .\end{cases}
$$

\subsection{Update of clock information}

Obviously, nodes $A$ and $B$ have their own latest clock skew at time of $t_{A}\left(t_{2}\right)$, while they exchange their own clock information table. For example, node $B$ has the latest clock skew of node $C$ in this cluster, node $A$ could update the clock skew of node $C$ $S_{C}(t)$ compared to the reference value; node $A$ updates this clock skew of node $C$ in its clock information table, then the relative clock skew between node $A$ and node $B$ is

$$
\left.S_{C}\left(t_{2}\right)=S_{C}(t)-S_{A}(t)\right) .
$$

Considering the reasonable topological structure, there can be effective reduction of energy consumption and communication interference, according to Radio Energy Dissipation Model (REDM), Load-balanced Hierarchical Topology Control Algorithm (LHTCA); the algorithm can effectively solve the failure of the cluster header, reduce WSN cluster-forming frequency, balance the energy consumption of network nodes, and thereby extend the network lifetime. On the basis of this, Hierarchical Wireless Sensor Network Management Protocol (HWSNMP) is well used for the design of WSN, and detailed descried WSN Management Information Base (MIB); HWSNMP had compact management object data type, protocol operation type as well as message format, and adopted Modified Encoding Rules (MER), which had higher coding efficiency than basic encoding rules, resulting in improved data acquisition and energy utilization rate.

\subsection{The execution of algorithm}

In order to verify the efficiency of the routing algorithm, we use $\mathrm{C}++$ to create the WSN simulation environment and compare to the routing algorithms which are researched recently in the two aspects of algorithm convergence speed and network 
lifetime. The results showed that the routing algorithm proposed here is superior to the current relatively new routing algorithms in convergence speed and network lifetime; it minimizes the WSN node's energy consumption and makes entire network load balance. Moreover, we combine WSN and the embedded technology, and design an environment information remote monitoring system based on WSN. According to the functional requirements of the monitoring system, the system will be designed in four modules which contain the terminal node, the coordinator node, the server and mobile terminal. For implementation of all the processors of terminal node and the coordinator node is used embedded chip CC2530; these two kinds of nodes use ZigBee technology to communicate with each other. WSN terminal nodes transmit the data to the coordinator nodes through the wireless network. The coordinator nodes will process the data and send it to the server side through GPRS module. We use socket technology to design the server side program. This program will process the data which is received from the coordinator nodes, then will store the data into database, and meanwhile process the request of mobile terminate. Mobile terminal's processor is implemented by embedded chip S3C2440A. The main function of mobile terminal is to collect environmental information and to display it to the user visually. The user can also send a query to the server in order to verify the feasibility of the system. It could bring great convenience to access the environmental information.

\section{Simulation}

In this section the performance of SRBC with PSR algorithm is compared. The two algorithms are implemented with different MAC protocols: RI-MAC, X-MAC and CSMA-MPS. The environment settings of the simulation are shown in Table 1.

Table 1. Simulation configuration
\begin{tabular}{|c|c|c|}
\hline Protocol & $\sigma_{\varphi}, \sigma_{\eta}, L, E_{\text {cal }}, E_{\text {com }}$ & Sample size \\
\hline RI-MAC & $15.3 \mu \mathrm{s}, 10^{-9}, 1 \mathrm{~ms}, 95.76 \mu \mathrm{J}, 160.68 \mu \mathrm{J}$ & 292 \\
\hline X-MAC & $1 \mathrm{~ms}, 10^{-9}, 7.5 \mathrm{~ms}, 95.76 \mu \mathrm{J}, 743.28 \mu \mathrm{J}$ & 454 \\
\hline CSMA-MPS & $1 \mathrm{~ms}, 10^{-9}, 7.5 \mathrm{~ms}, 95.76 \mu \mathrm{J}, 743.28 \mu \mathrm{J}$ & 398 \\
\hline
\end{tabular}

As shown in Fig. 2, in RI-MAC protocol, the energy consumptions per rendezvous have similar substantial increase for the two algorithms with the increase of the communication density; SRBC can significantly reduce the energy consumption compared to PSR. As shown in Fig. 3, the increasing ratio of the communication consumption in X-MAC protocol decreases with the increase of the communication density; SRBC has the more energy saving than PSR. In Fig. 4, the performance in the CSMA-MPS, SRBC could save more energy than PSR since the sharing of the clock information, so it improves the performance in different communication traffic as well as different MAC protocols. 


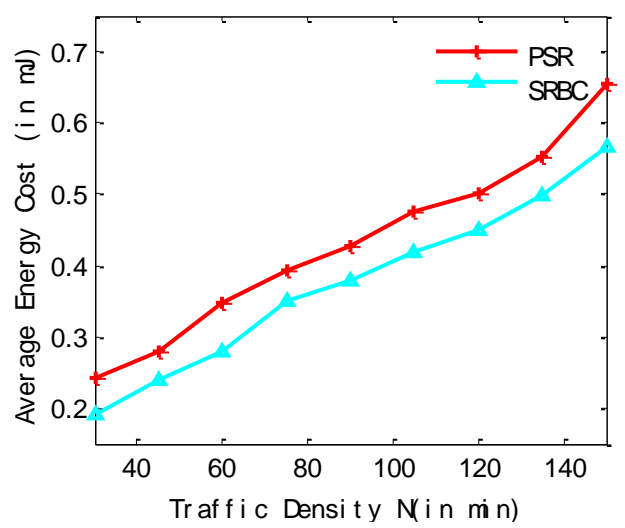

Fig. 2. Average energy cost in RI-MAC

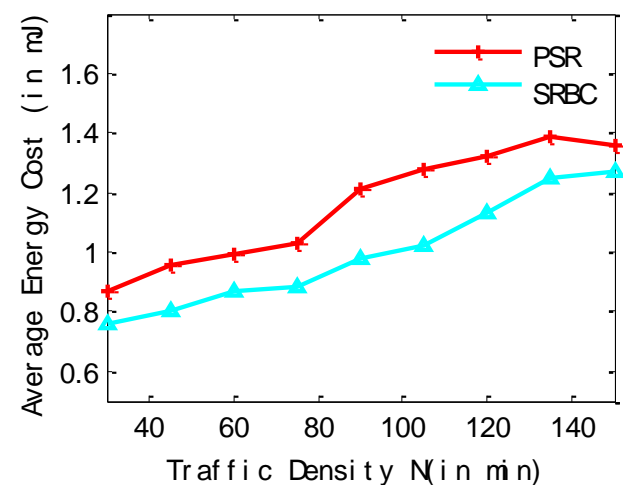

Fig. 3. Average energy cost in X-MAC

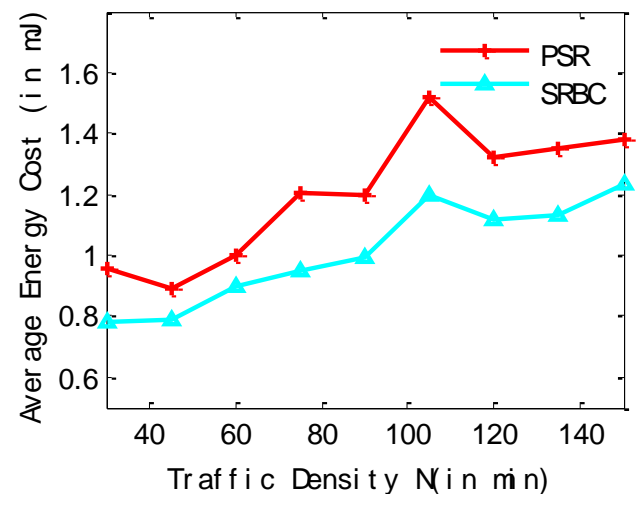

Fig. 4. Average energy cost in CSMA-MPS

\section{Conclusion}

This paper presents SRBC algorithm in low-duty-cycle WSN for the synchronous rendezvous. This paper focuses on improving the performance of synchronous 
rendezvous in the low-duty-cycle wireless sensor network with the existence of relative clock skew in each node. The algorithm improves the effectiveness and the reliability of the synchronous rendezvous trough the sharing of clock information in a cluster as well as among the clusters, to reduce estimated clock error based on the normal communication. In practical applications, the nodes' work time obey some probability distributions, and the algorithm would achieve greater performance if combined with these distributions.

Acknowledgments: This work is supported by the Natural Science Foundation Project of CQ CSTC: $2011 \mathrm{BB} 2064$.

\section{References}

1. Chen, L.-Y. et al. Scheduling Scheme Algorithm in Low-Duty-Cycle WSN. - Journal of Software, 2014, No 03, pp. 631-641.

2. H i 11, J. L., D. E. C u 11 e r. MICA: A Wireless Platform for Deeply Embedded Networks. - Micro IEEE, Vol. 22, 2002, No 6, pp. 12-24.

3. D u t t a, P., D. C u 11 e r. Practical Asynchronous Neighbour Discovery and Rendezvous for Mobile Sensing Applications. - Sensys'08, pp. 71-84.

4. Hu ang, D.-J. et al. A Defense against Clock Skew Replication Attacks in Wireless Sensor Networks. - Journal of Network and Computer Applications, Vol. 39, 2014, No 3, pp. 26-37.

5. B u et tn e r, M., G. V. Y e e, E. Anderson et al. X-mac: A Short Preamble Mac Protocol for Duty-Cycled Wireless Sensor Networks. - Sensys, 2006, pp. 307-320.

6. S u n, Y., O. Gu r e wit z, D. B. John so n. RI-MAC: A Receiver-Initiated Asynchronous Duty Cycle MAC Protocol for Dynamic Traffic Loads in Wireless Sensor Networks. - In: Proc. of ACM SenSys, 2008, pp. 1-14.

7. Ye, W., F. S ilva, J. He i d e mann. Ultra-Low Duty Cycle MAC with Scheduled Channel Polling. - SenSys'06, pp. 321-334.

8. Ganeriwal, S., I. Ts igkogiannis, H. Si m, V. Tsiatsis, M. B. Srivastava, D. Ganesan. Estimating Clock Uncertainty for Efficient Duty-Cycling in Sensor Networks. - Networking on IEEE/ACM Transactions, Vol. 17, 2009, No 3, pp. 43-56.

9. Ch e n g, L., Y. G u, T. H e, J. N i u, L. Ch e n g, Y. G u et al. Dynamic Switching-Based Reliable Flooding in Low-Duty-Cycle Wireless Sensor Networks. - Proc. of INFOCOM, IEEE, Vol. 12, 2013, No 11, pp. 1393-1401.

10. H a o, H., J. Y u n, Z. Z h o n g, S. K i m, T. H e. PSR: Practical Synchronous Rendezvous in LowDuty-Cycle Wireless Network. - Proc. of Infocom, IEEE, Vol. 12, 2013, No 11, pp. 2661-2669. 